\title{
Bioethical aspects in the approach of the minor patient - general review
}

\author{
Andreea Salcudean ${ }^{1}$, Monica Kiss ${ }^{2}$, Cristina Raluca Bodo ${ }^{3}$, \\ Maria Melania Lica', Elena Gabriela Strete ${ }^{1}$ \\ 1 "George Emil Palade" University of Medicine, Pharmacy, Sciences and Technology, \\ Targu Mures, Romania \\ 2 Psychiatry Department, City Hospital, Odorheiu Secuiesc, Romania \\ ${ }^{3}$ Psychiatry Clinic 2, County Clinical Hospital Mures, Targu Mures, Romania
}

\begin{abstract}
The objective of this review was to present the main ethical issues in minor patient healthcare activity including: minor patient consent, parental consent, confidentiality and medical examination. The particular ethical issues that arise in the medical care of children and adolescents overlap with those of other patients, who lack decision-making capacity or have diminished decision-making capacity. Children should always be respected as persons. A consent from the child and parental permission be sought whenever possible, by using appropriate tools. Even minor patient demonstrated the ability to understand essential elements of healthcare activity and the details of the medical examination, there is variability in children's level of understanding, mainly due to their age and maturity. The legal framework in every country represents a guide for the medical doctors and a reference for their patients.
\end{abstract}

Keywords: autonomy, confidentiality, consent, capacity, minors, health care decision

\section{INTRODUCTION}

The particular, ethical problems that arise in the medical care of children and adolescents overlap with those of other patients, who lack decisionmaking capacity or have a diminished decisionmaking capacity. However, there are differences. In children case, the presence of a decision-making capacity, the role of the child patient's own values and the distinct role of the surrogate decision-makers, the parents. The relations with a child patient is loaded with a lot of tension. It is very important to value intra-family relationships, giving parents the right to make decisions regarding the care of their children without other external interventions. The medical doctor has a duty to protect the child from what is bad, if the parent does not protect the child patient interests [1].

Principles of biomedical ethics were proposed by Beauchamp and James Childress in an effort to resolve ethical issues in clinical medicine. In both clinical medicine and in scientific research these principles can be applied, even in unique circumstances, to provide guidance in discovering our moral duties within that situation. These principles are presented in Table 1.

TABLE 1. Ethical principles and professional obligations (Beuachamp and Childress) [2]

\begin{tabular}{|l|l|}
\hline Ethical principles & Professional obligations \\
\hline Beneficence & Professional secrecy \\
\hline Non-maleficence & Faithfulness \\
\hline Autonomy & Implication \\
\hline Justice & Truthfulness \\
\hline
\end{tabular}

\section{INFORMATION AND CONSENT IN PEDIATRICS - PARTICULAR ASPECTS}

Western society is offering a strong emphasis on self-determination and patients' rights. The model of autonomy and decision-making is operationalized in the informed consent processes outlined in common law by multiple forms of health care legislation [3].

As well known in literature, informed consent requires that a decision maker: has mental capacity for 
making the decision, is adequately informed, that all relevant information is given, and the decision witch results must be voluntary and free of coercion [4].

The first and the most difficult process is to determine if the child patient is having or not mental capacity. The child patient may be able to understand the treatment decision information and even to appreciate reasonably the consequences of the decision or the lack of it [5].

In the case of a minor patient, his mental capacity is either lacking, either difficult to determine and it depends on the age, self maturity and on specific circumstances.

The most appropriate substitute decision-makers for infants and young children are parents and legal guardians [6].

The capacity of the minor patient to consent or to refuse to a proposed treatment varies with age and cognitive capacity is determined on a case-by-case basis. The target is to ensure that the resultant decision is voluntary and free of any coercion. When making treatment decisions for a minor patient, the primary concerns of doctors and parents should be to maximize benefits and minimize harm [4].

Competent adults, able to understand the occurring transformations, must respond to the child's competences. Young adolescents are aware of the severity of their disease and the treatment importance [2].

The information provided to the minor patient is different from that provided to an adult patient and is adapted to the child's age and the specific aspects involved in the information. The child, affected by his disease, suffering, may not have the same ability to understand and communicate the same as a healthy child of the same age may do.

The first step in the healthcare process is the doctor's assessment of a minor patient's capacity to understand the particular circumstances relevant to his illness and of the medical treatment. No universally acceptable, validated tool exists in the paediatric world to determine the minor patient capacity [7].

In this sense, there are some particular aspects that support the medical approach: recognition of the stages of development of the child's cognitive capacity; accepting and respecting the emotional status of the child throughout the hospitalization; accepting the family as a partner in the therapeutic process, each member of the family having a well-established place;

When transmitting medical information to a child the medical doctor must consider several principles: when the new medical information is offered to a minor patient, the patient must be seen as a bio-psycho- social unit in a socio-economic, cultural and family context; when we transmit information to a child we must not evoke other levels of responsibility otherwise the child can blame himself instead of becoming responsible; a method of making the minor patient responsible is to involve him like an actor in the theater of the events he is going to live [8].

Children, due to the fact they cannot think and act like adults, by their nature are considered unable to consent. This is real in the case of very young children, but once the maturation process begins, individual differences appear. The newborn and children under the age of 4 cannot make decisions, do not have the ability to consent, so those who are able to do so, are their parents, who naturally should decide what is best for their child. There are also situations in which, through their decision, they can bring major damage to the health of their children. Therefore, most states have regulations designed to protect the child if the parent is either unable or unwilling to make a decision aimed to assure the absolute good. There is no fixed chronological age that can establish the child's competence due to the heterogeneity of personal development factors and socio-familial situation.

Medical decision-making in adolescents is very complicated. Some adolescents may demonstrate decision-making capacity to adults in empirical studies [6], their ability to make appropriate decisions is affected, and maybe impaired by different factors, such as psychosocial context, peer pressure, impulsivity and some risk-seeking pathological traits of behavior [9].

Romanian law Family Code stipulates that parents have the right and duty to represent their minor child in the activities of the minor until he reaches the age of 14. In Romania, the competence or capacity to exercise is accepted to be acquired at the age of 18 and allow the young adult to exercise his rights and assume his obligations through legal acts [10].

The discernment should not be seen as the capacity of exercise but as a premise of it. The presence or absence of discernment are aspects that can characterize a person regardless of age, while the capacity to exercise, as a legal term, is acquired at a certain age. There are three forms of capacity of exercise, from a legal point of view: lack capacity of exercise, reduced capacity of exercise and full capacity of exercise. For minors aged 14-18 years, it is a matter of reduced capacity and the conclusion of legal acts is done only with the consent of the legal guardian [10].

With regard to the minor patient, who does not have the capacity to consent, it must be emphasized that no intervention will be made without the consent 
of his legal representative or another person designated by law. In Romania, the law sets the age for expressing informed consent at 18 years. In emergency situations, the minor with discernment, in the absence of the legal representative or of the parents, can express his consent after the age of 14 years. Regarding the medical situations related to the diagnosis and treatment of sexual and reproductive problems, the minor patient over 16 years of age can give his consent.

In emergency situations, when the minor's life is endangered and the rescue intervention is imminent, neither the response of the guardianship authority nor of the legal representative is expected.

A general available guidance scheme adapted [1] is presented in Figure 1.

\section{CONFIDENTIALITY IN THE CASE OF MINOR PATIENTS}

The medical doctor must receive consent before disclosing confidential information, unless this wo- uld slow down the transmission of information (which would increase the risk of harm to the minor patient), or seek consent would endanger the minor or others. If a young person reports abuse, it is mandatory to explain him that the doctor must report the event, this being an exception from confidentiality. Confidential information may be disclosed without approval if required by law or if the benefits of disclosing information about a minor outweigh the interests of the individual (and the public) in maintaining confidentiality. Disclosed information must be essential, relevant, accurate and secure. Information sharing decisions (or not) should be explicitly documented [1].

There is a great challenges for medical doctors covering all the aspects related to the variability in laws and policies, understanding the limits of confidentiality related to their practice, concerning the confidentiality aspects related to their patients. In a study regarding the minor patient's risky behaviors, defined as health compromising behaviors pertaining to smoking, alcohol use, drug use, sexual behavior, and sui-

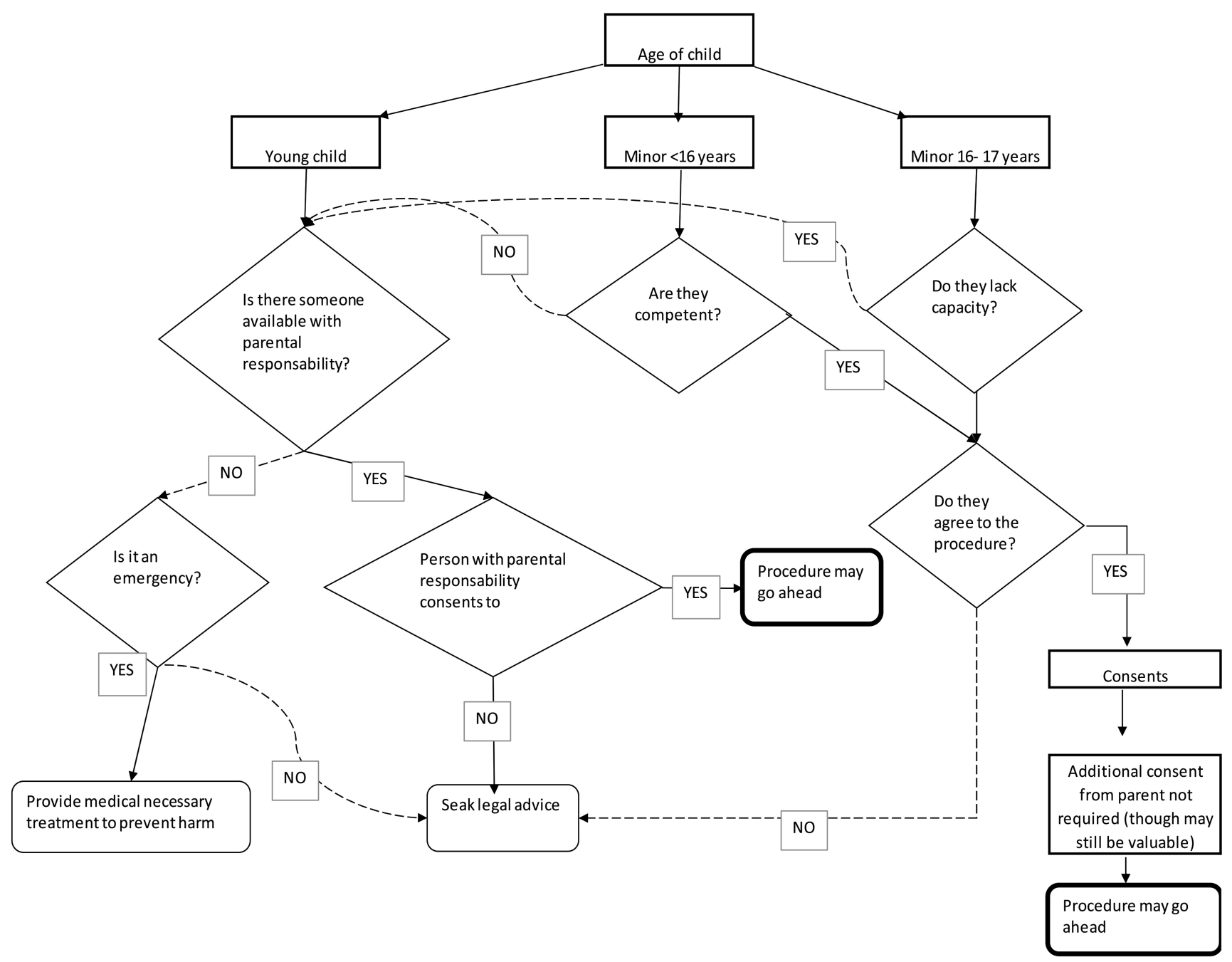


cidal behavior, researchers found that healthcare providers feel ethically obligated to break confidentiality and inform minor patient's parents of these behaviors, if they consider it would result in unfavorable outcomes to either the patient or others. Physician's professional judgment is critical in determining the point at which the behavior becomes a significant threat [11].

It should be recognized that robust confidentiality protection for minor patients will require coordination among multiple stakeholders beyond the level of individual providers or institutions. Legislative advocacy efforts are ongoing present on issues regarding mandatory reporting of "statutory rape," consent provisions for alcohol/drug abuse, mental health, and reproductive health services, mature minor statutes, and "health care emancipation" laws [12].

The literature sustains that adolescents who are assured of confidentiality by a physician that afterwards reported greater willingness to disclose sensitive information like mental health problems, sexual history substance abuse, will not seek future health care, as compared to those for whom confidentiality was not disclosed $[13,14]$.

\section{MEDICAL EXAMINATION AND APPROACH OF THE MINOR PATIENT IN TERMS OF THE PARTICULARITIES OF THE DISEASE}

Doctors may be asked by local authorities or other doctors to perform an assessment. They should only accept if they have the basic skills (abilities, knowledge) needed for this. If a medical examination is requested, consent should be obtained from the minor patient if he or she is 16 or 17 years, or from a parent or caregiver. If the parent refuses an examination that the doctor deems it necessary, or the minor patient refuses to give his consent, the doctor should discuss and ask for the consent of the guardianship authority [1].

Even minor patient demonstrated the ability to understand essential elements of healthcare activity and the details of the medical examination, there is variability in children's level of understanding, mainly due to their age and maturity.

The legal framework in every country represents a guide for the medical doctors and a reference for their patients.

Of great significance in medical practice, which most doctors face, is the transmission of a diagnosis with a reserved prognosis. For such situations, protocols have been developed at the hospital level, which provide for the way in which the patient or family, can give their consent or can refuse medical treat- ments or investigations or can ask for a second medical opinion.

The medical branches most often confronted with such hypostases are: oncology, psychiatry, cardiology, gastroenterology, etc. Bioethicists are the ones who make the proposal to take into account the protocol that informs patients about serious suffering, the transmission to the patient and family of complete medical information for everyone to understand, the provision of psychological support and the involvement of the patient and family.

One important concern of in current pediatric is the psychiatric diagnosis where is present the issue of stigma, so from here appear obvious the need to classify diseases as a codified form of communication, between specialists [15]. All this comes in the interest of the minor patient, because he can slip on the slope of inaccurate labels that certainly in addition to the basic diagnosis can affect the quality of his life and limit his future prospects.

In addition to drug treatment, individual and family psychotherapy are probably the most common forms of treatment in child psychiatry [15].

The psychotherapist faces ethical issues related to the questionable benefits and ongoing training programs of the therapist, so as to step out of the field of scientifically outdated and unfounded techniques, and children and parents must be properly informed of how essential therapy is and its inclusion, starting early as the assessment stage.

All these aspects are a necessity that comes in both the emotional and physical support of the child with the involvement of his family and to transcend the boundaries that include ethical issues. This optimally ensures the fundamental needs on which each patient depends in order to benefit from an increase in the quality of life even under the influence of diseases, professionally managed by medical staff regardless of specialty.

Ethical problems involving children and their health are in a more increasing attention $[16,17]$.

\section{CONCLUSIONS}

In any health problems regarding children, it is important to consider how the ethical issues should be solved, and to document into practices.

Children should always be respected as persons, a consent from the child and parental permission should be sought whenever possible, by using appropriate tools. Special attention has to be paid to the most vulnerable children (institutionalised, impoverished, homeless, and sick children). Doctors should assess child's mental capacity before involving in a therapeutic decision. 
Children from different countries or being from different, social, religious, ethnic groups should

\section{REFERENCES}

1. Herring J, Wilkinson D, Savulescu J. Medical ethics and law: a curriculum for the 21st century. Third edition. Edinburgh: Elsevier, 2020.

2. Buta MG, Buta L. Bioetica in pediatrie. Cluj Napoca: Editura Eikon, 2008.

3. Osborne PH. Consent to treatment. In: Irvine JC, Osborne PH, Shariff M (eds.). Canadian Medical Law: An Introduction for Physicians, Nurses and Other Health Care Professionals. Toronto, Ont: Thomson Reuters, 2013:34.

4. Coughlin KW. Medical decision-making in paediatrics: Infancy to adolescence. Paediatrics \& Child Health. 2018 Apr 12;23(2):138-46.

5. Etchells E, Sharpe G, Elliott C, Singer PA. Bioethics for clinicians: 3 . Capacity. CMAJ. 1996 Sep 15;155(6):657-61.

6. Harrison C, Kenny NP, Sidarous M, Rowell M. Bioethics for clinicians: 9. Involving children in medical decisions. CMAJ. 1997;156(6):825-8.

7. Raymundo MM, Goldim JR. Moral-psychological development related to the capacity of adolescents and elderly patients to consent. J Med Ethics. 2008 Aug;34(8):602-5.

8. Hirsch $E$. Quelques repères pour une éthique de la communication et de l'information en pédiatrie. Archives de Pédiatrie 2000; 7(2):153s-155s.

9. Diekema DS. Adolescent refusal of lifesaving treatment: are we asking the right questions? Adolesc Med State Art Rev. 2011 Aug;22(2):213-28, viii. always be treated with the same respect and consideration.

\section{Conflict of interest: none declared Financial support: none declared}

10. Reghini I, Diaconescu S. Introducere în dreptul civil, vol. I. Cluj Napoca: Editura Sfera Juridică, 2004.

11. Rae WA, Sullivan JR, Razo NP, George CA, Ramirez E. Adolescent health risk behavior: when do pediatric psychologists break confidentiality? J Pediatr Psychol. 2002 Sep;27(6):541-9.

12. Pathak PR, Chou A. Confidential Care for Adolescents in the U.S. Health Care System. J Patient Cent Res Rev. 2019;6(1):46-50.

13. Ford CA, Thomsen SL, Compton B. Adolescents' interpretations of conditional confidentiality assurances. J Adolesc Health. 2001 Sep;29(3):156-9.

14. Ford CA, Millstein SG, Halpern-Felsher BL, Irwin CE. Influence of physician confidentiality assurances on adolescents' willingness to disclose information and seek future health care. A randomized controlled trial. JAMA. 1997 Sep 24;278(12):1029-34.

15. Bloch S, Chodoff P, editors. Psychiatric ethics. 2nd ed. Oxford ; New York: Oxford University Press; 1991.

16. Van Larebeke NA, Birnbaum LS, Boogaerts MA, Bracke M, Davis DL, Demarini DM, et al. Unrecognized or potential risk factors for childhood cancer. Int J Occup Environ Health. 2005 Jun; 11(2):199-201.

17. Gilbert SG. Ethical, legal, and social issues: our children's future. Neurotoxicology. 2005 Aug;26(4):521-30. 